\title{
The Commandable Rate Scanner: Precision Attitude Sensing for Spinning Spacecraft
}

\author{
E. David Skulsky ${ }^{\dagger}$ \\ Microcosm, Inc., Torrance, California 90505
}

\begin{abstract}
High performance Earth sensing for spin-stabilized vehicles can be achieved through a straightforward modification to a flight-proven conical Earth horizon sensor. The necessary hardware and, therefore, cost and weight, is essentially identical to hardware used by three-axis stabilized satellites, making precision attitude sensing available to the entire spacecraft community, including small satellite builders. The Barnes Engineering Commandable Rate Scanner uses a rotating sensor on a spinning spacecraft to provide full-sky ( $4 \pi$ steradian) Earth coverage with a single sensor. The sensor provides data for any spacecraft attitude and altitude from LEO to above GEO. In LEO, a data rate of several hundred horizon crossings per minute is typical, allowing for extremely accurate (better than $0.02 \mathrm{deg}$ ) attitude sensing. In addition, the principal biases which normally dominate attitude determination accuracy for spinning spacecraft are either eliminated or can be measured. Computational complexity is low, and example algorithms for sensor data processing are provided. Simulation results show that the sensor performs well even in the presence of substantial spacecraft nutation.
\end{abstract}

TABLE OF SYMBOLS

a.................Azimuth of Horizon Crossing Vector $\delta$..................Elevation of Horizon Crossing Vector $\gamma$.................................... Sensor Half-Cone Angle $\rho$......................................... Earth Angular Radius

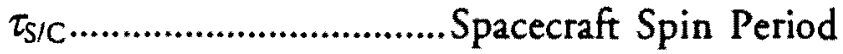
$\tau_{\text {sensor }}$..........................................Sensor Spin Period $\omega_{S / C}$.......................................Spacecraft Spin Rate $\omega_{\text {sensor }}$........................................Sensor Spin Rate Alt ..................................... Spacecraft Altitude h ................................. Horizon Crossing Vector H ................ Matrix of Horizon Crossing Vectors $M$..................................... B Base Multiplier n ......................................... Unit Nadir Vector N...................................umber of Measurements $P$...................................... ORS Offet Step Size $R_{E} \ldots \ldots . .$. Earth Equatorial Radius $(=6378.14 \mathrm{~km})$

"Funded, in part, by the Naval Research Laboratory. †Systems Engineer.
$S R$ $t_{H C}$ Sensor-to-Spacecraft Spin Ratio ..Horizon Crossing Time

\section{INTRODUCTION}

Earth-referenced attitude sensing on spinning satellites is usually performed by one or more Horizon Crossing Indicators ( $\mathrm{HCI})$. The $\mathrm{HCI}$ is simple, reliable, and reasonably priced. Nevertheless, it does have drawbacks. First, the $\mathrm{HCI}$ is only modestly accurate. Even under the best of circumstances, accuracy of $0.1^{\circ}$ is difficult to achieve [ref. 1]. Second, altitude and attitude operating restrictions often limit when and how the $\mathrm{HCI}$ can be used. Steerable $\mathrm{HCl}$ s can alleviate this problem by adjusting the half-cone angle in orbit, but this requires ground commanding. Third, the $\mathrm{HCI}$ is sensitive to mounting and altitude biases. A bias in the sensor half-cone angle, a principal axis misalignment, or an error in the spacecraft altitude directly affect sensor accuracy. Finally, the $\mathrm{HCI}$ functions poorly in the presence of even moderate spacecraft nutation. 
As a consequence of these difficulties with the $\mathrm{HCI}$, the sensor is generally not used for attitude determination when moderate to high accuracy sensing is required. Instead, star sensing or another high-accuracy attitude sensing technique must be used. Horizon crossing indicators are often flown simply for control purposes during a small segment of a mission (e.g. during transfer to operational orbit), wasting valuable spacecraft real estate and mass. This paper describes a new approach to Earth sensing for spinning satellites which promises far greater accuracy, bias insensitivity or detectability, and excellent performance in the presence of substantial nutation. In addition, this approach provides $4 \pi$ steradian (full-sky) Earth detection, functions well from LEO to GEO and beyond, has low computational complexity, can be configured for autonomous navigation, and utilizes a sensor with substantial flight heritage.

\section{CRS OPERATIONS CONCEPT}

The Barnes Engineering Commandable Rate Scanner is a rotating sensor with a $90^{\circ}$ half-cone angle. On a three-axis stabilized spacecraft, the sensor field of view sweeps out a plane in the sensor sky. Since the sensor scan axis is fixed with respect to the spacecraft, the sensor continually scans the same points in the spacecraft sky. However, on a spinning vehicle, the vehicle motion moves the sensor spin axis. As shown in fig. 1, the sensor spin axis is mounted in the spacecraft spin plane so that the sensor field of view (FOV) undergoes a complex, albeit well-defined, motion which is a combination of the motion of the sensor and the motion of the spacecraft. As described below, the motion of the FOV is uniquely determined by the sensor-to-spacecraft spin ratio, $S R$. Throughout this paper we will refer to an example case of a 20 rpm spacecraft and a sensor which spins at about $240 \mathrm{rpm}$, producing an $S R$ of approximately 12 .

Figure 2 illustrates the basic concept of

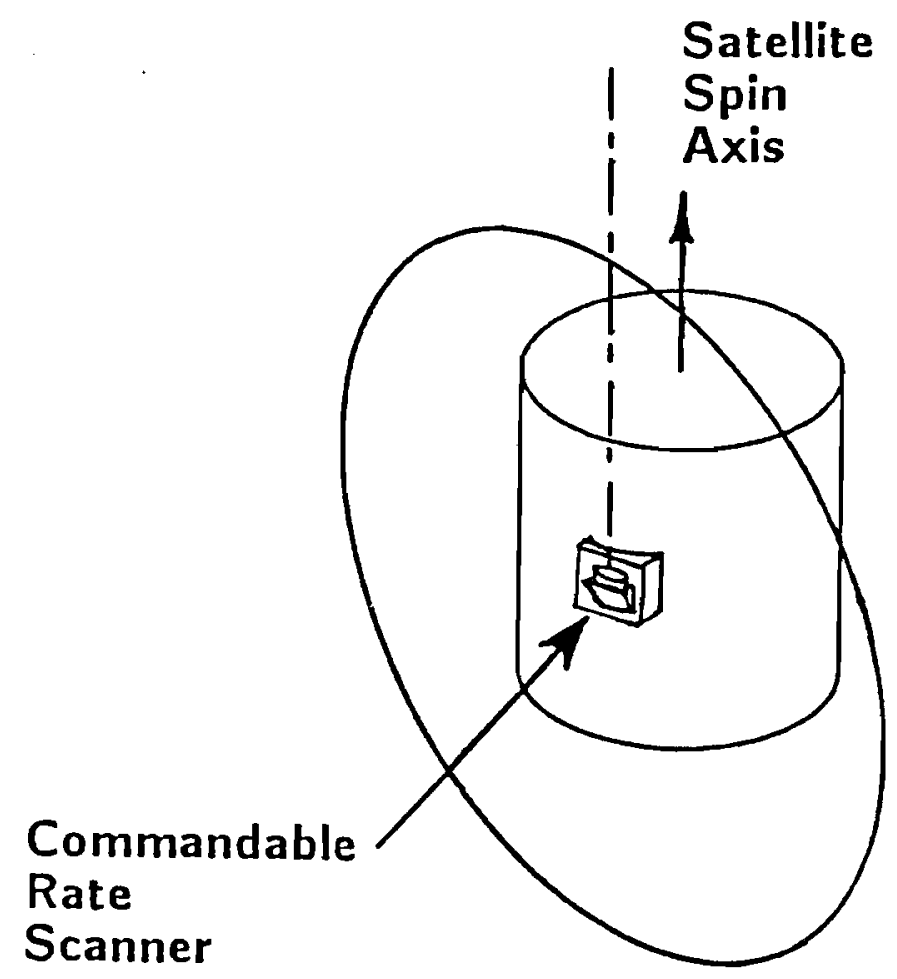

Figure 1: Barnes Engineering Commandable Rate Scanner on Spacecraft 
operation for a rotating sensor on a spinning spacecraft. The sphere in this figure is the inertially fixed spacecraft-centered celestial sphere-the sphere is not rotating with the spacecraft. Here we have traced out the path of the sensor FOV during approximately one sensor revolution. The analemma, or "figure $8, "$ is the shape of the sensor path in inertial space when $S R \cong$ 1. Note that when the FOV crosses the spacecraft spin plane, the angle between the path of the FOV and the spacecraft spin plane will be approximately $45^{\circ}$, corresponding to equal vertical (due to the motion of the sensor) and horizontal (due to the motion of the spacecraft) components. If $S R \equiv 1$, the analemma will close. Otherwise, the analemma will precess around the celestial sphere until the entire sky is covered.

As $S R$ increases, the FOV path becomes vertical and more densely covers the spacecraft sky, as illustrated in fig. 3. Here $S R=6$, so the angle between the path of the FOV and the spacecraft spin plane will be $\arctan (6)=80.5^{\circ}$.

\section{Effect of Sensor-to-Spacecraft Spin Ratio}

$S R$ selection is based on a number of criteria, including the spacecraft spin rate, the nominal sensor spin rate, and Earth geometry, but an important point which must be considered when choosing $S R$ is that the FOV path will repeat exactly after a single spacecraft revolution when $S R$

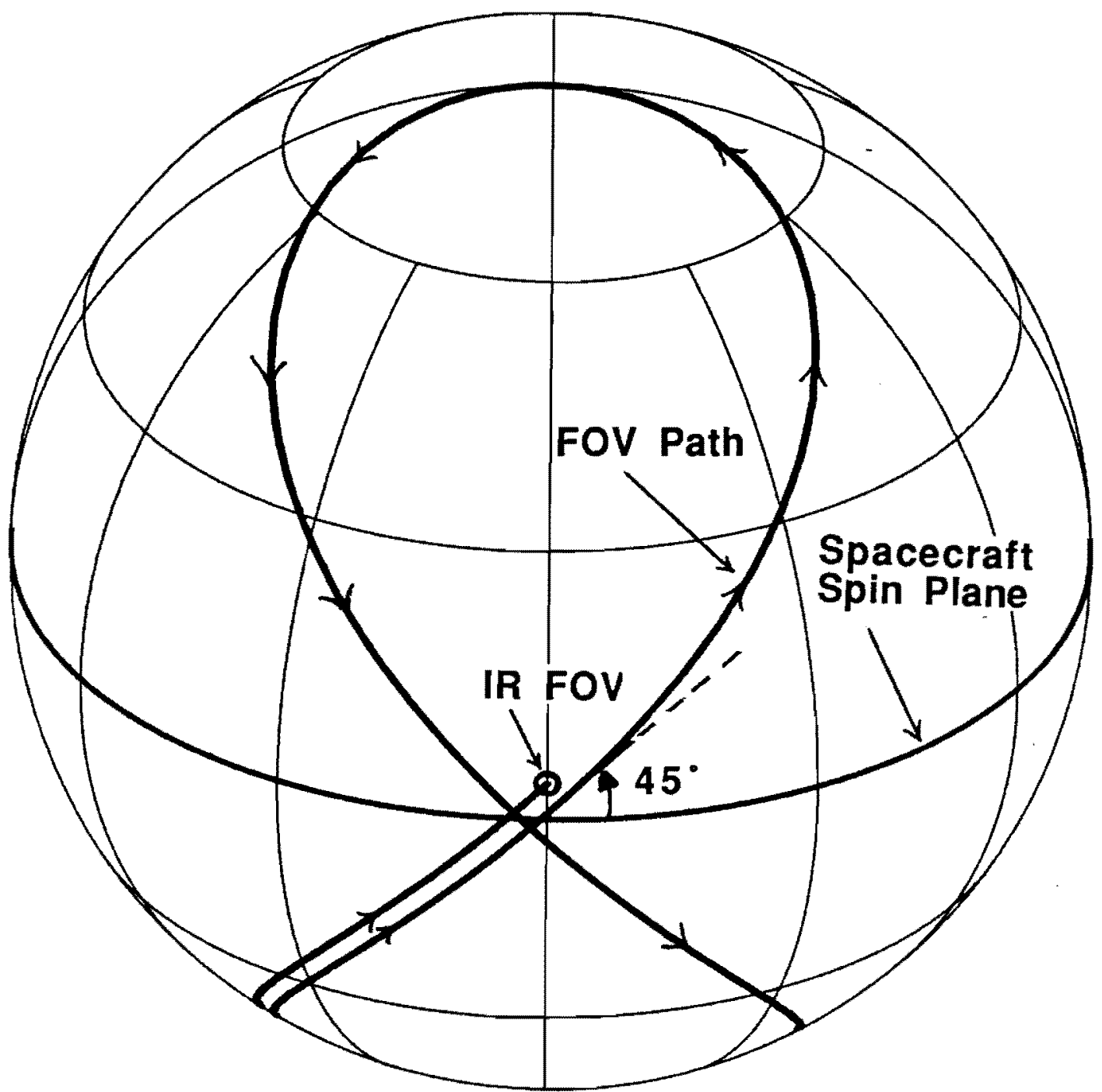

Figure 2: FOV Path of a Rotating Sensor on a Spinning Spacecraft [SR $\cong 1]$

(Spacecraft-Centered Celestial Sphere). 
is an integral number. If $S R$ is a rational nonintegral number, the FOV path will repeat, but only after multiple spacecraft revolutions. Figures 3 and 4 show the FOV path over several spacecraft revolutions for $S R=6$ and $S R=6.1$, respectively.

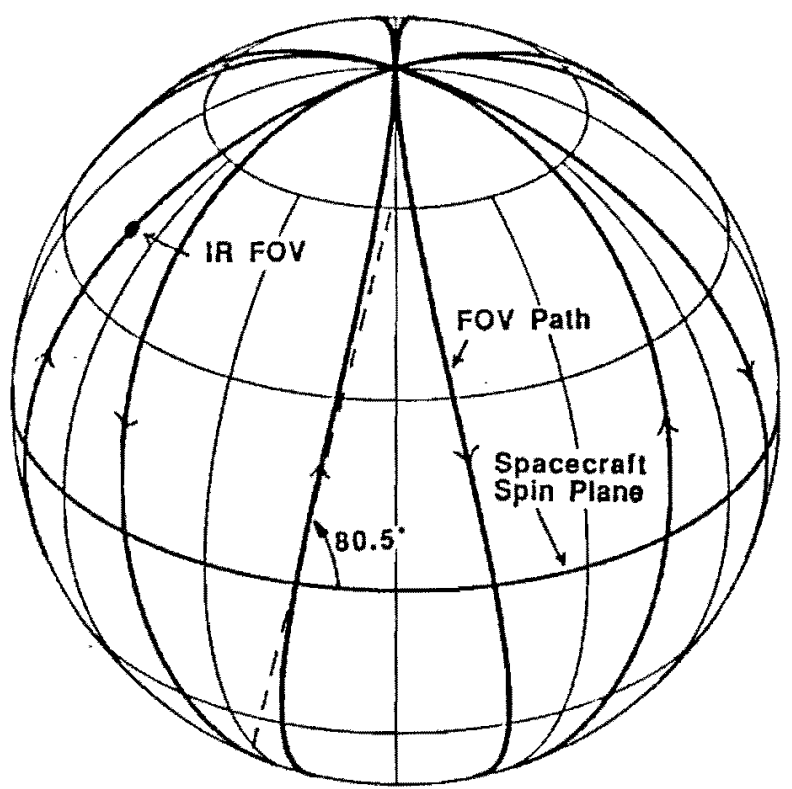

Figure 3: CRS FOV Path for $S R=6$.

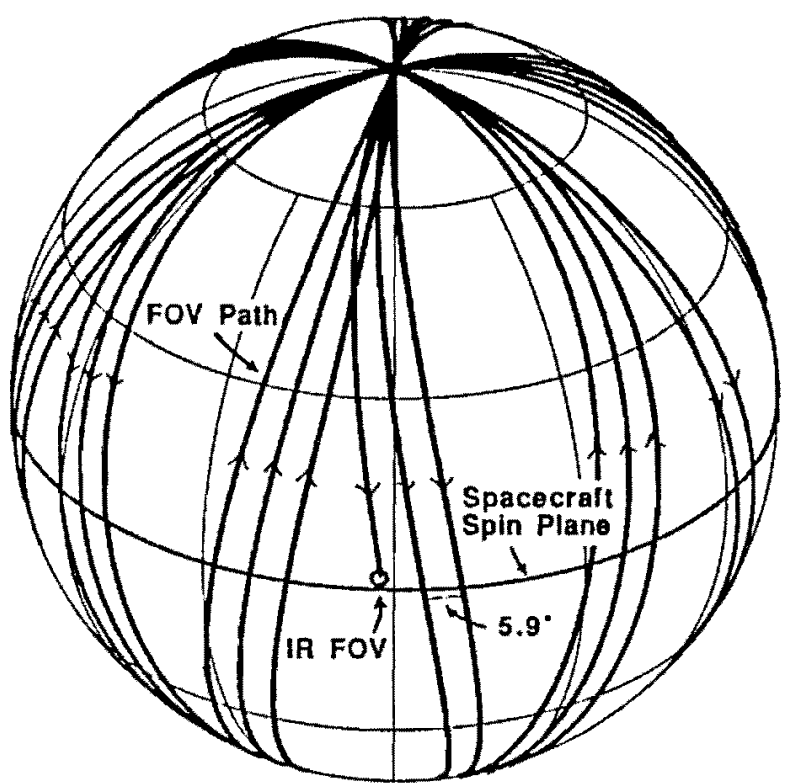

Figure 4: CRS FOV Path for $S R=6.1$.

Control of the sensor-to-spacecraft spin ratio, $S R$, is critical to the operation of the CRS. It is convenient here to think of $S R$ as composed of two terms: the base multiplier and the offset step size.
The base multiplier, $M$, is an integer multiple of the spacecraft spin rate which determines the general character of the sensor scan pattern. The offset step size, $P$, is the value by which the scan pattern precesses for each spacecraft revolution. In other words, if we would like the spin plane crossing point to precess by $P$ degrees every spacecraft revolution, then we can compute $S R$ as follows:

$$
S R=\left(\frac{360^{\circ}}{360^{\circ}-P}\right) M
$$

For example, if $S R=6.1$ (implying $M=6$ ), we get $P=5.9^{\circ}$. Figure 4 shows the scan pattern and spin plane crossing point precession for this case. The sensor FOV will return to its starting position after $360^{\circ} / 5.9^{\circ}=61$ spacecraft revolutions. By making small adjustments to the sensor scan rate, the precession can be made either positive or negative and can be as large or fine as necessary.

\section{Data Rates}

Attitude determination accuracy is strongly affected by the number of horizon crossings obtained for the period of interest. Table 1 lists the data rates that will be obtained for a spacecraft spinning at $20 \mathrm{rpm}$ versus various sensor scan rates. The maximum number of horizon crossings will be obtained when the spacecraft spin axis is pointing in vicinity of nadir, regardless of the spacecraft altitude. The minimum number of crossings shown is for a LEO spacecraft when the spacecraft spin axis is $90^{\circ}$ from nadir.

\begin{tabular}{|c|c|c|}
\hline $\begin{array}{c}\text { CRS scan } \\
\text { rate } \\
\text { (rpm) }\end{array}$ & $\begin{array}{c}\text { Min. \# of } \\
\text { crossings per } \\
\text { S/C rev. }\end{array}$ & $\begin{array}{c}\text { Max. \# of } \\
\text { crossings per } \\
\text { S/C rev. }\end{array}$ \\
\hline 240 & 16 & 24 \\
\hline 120 & 8 & 12 \\
\hline 60 & 4 & 6 \\
\hline
\end{tabular}

Table 1: Earth data rate for $20 \mathrm{rpm}$ spacecraft at $1100 \mathrm{~km}$ altitude.

Horizon crossing indicators provide either zero or two crossings per spacecraft revolution, 
depending on the orientation of the spacecraft spin axis. The CRS always provides more than two crossings, even when the sensor is spinning well below the recommended rate.

\section{HARDWARE}

The Commandable Rate Scanner traces its heritage to over 500 scanning infrared sensors and uses essentially the same sensor head flown on the Cosmic Background Explorer (COBE) mission-a $90^{\circ}$ half-cone angle with a nominal spin rate of 240 $\mathrm{rpm}(4 \mathrm{~Hz})$. The electronics, however, differ from the COBE flight unit (and all other standard conical Earth sensors, for that matter) in the following ways: the CRS provides the time of each horizon crossing, rather than the phase with respect to an internal reference; the sensor scan rate must be variable; and the satellite spin rate must be obtained from Sun pulses provided by an external sensor or some other method. Rather than modify the COBE electronics, a more flexible processing unit will be adapted. The system employs a 1750 microprocessor and provides 20-bit serial words over a MIL-STD-1553 data bus.

An example schematic of the CRS motor speed control system is shown in fig. 5. The lower portion of the figure is a phase-locked loop (PLL) which keeps the motor speed constant according to an input reference signal. An optical phase reference pickup (PRP) produces 72 pulses for each sensor revolution (every $5^{\circ}$ ). At about 4 revolutions per second, the PRP produces a signal of approximately $288 \mathrm{~Hz}$. This approach has been found to keep flutter within each $5^{\circ}$ encoder interval to less than $0.03^{\circ}(3 \sigma)$.

The PLL reference signal is generated by the circuit shown in the upper portion of fig. 5. This signal will be adjusted to keep the Sensor-toSpacecraft Spin Ratio constant, based on spacecraft spin rate measurements. Another phase-locked loop generates a signal of frequency 900 times the spacecraft spin rate (as sensed using Sun pulses).

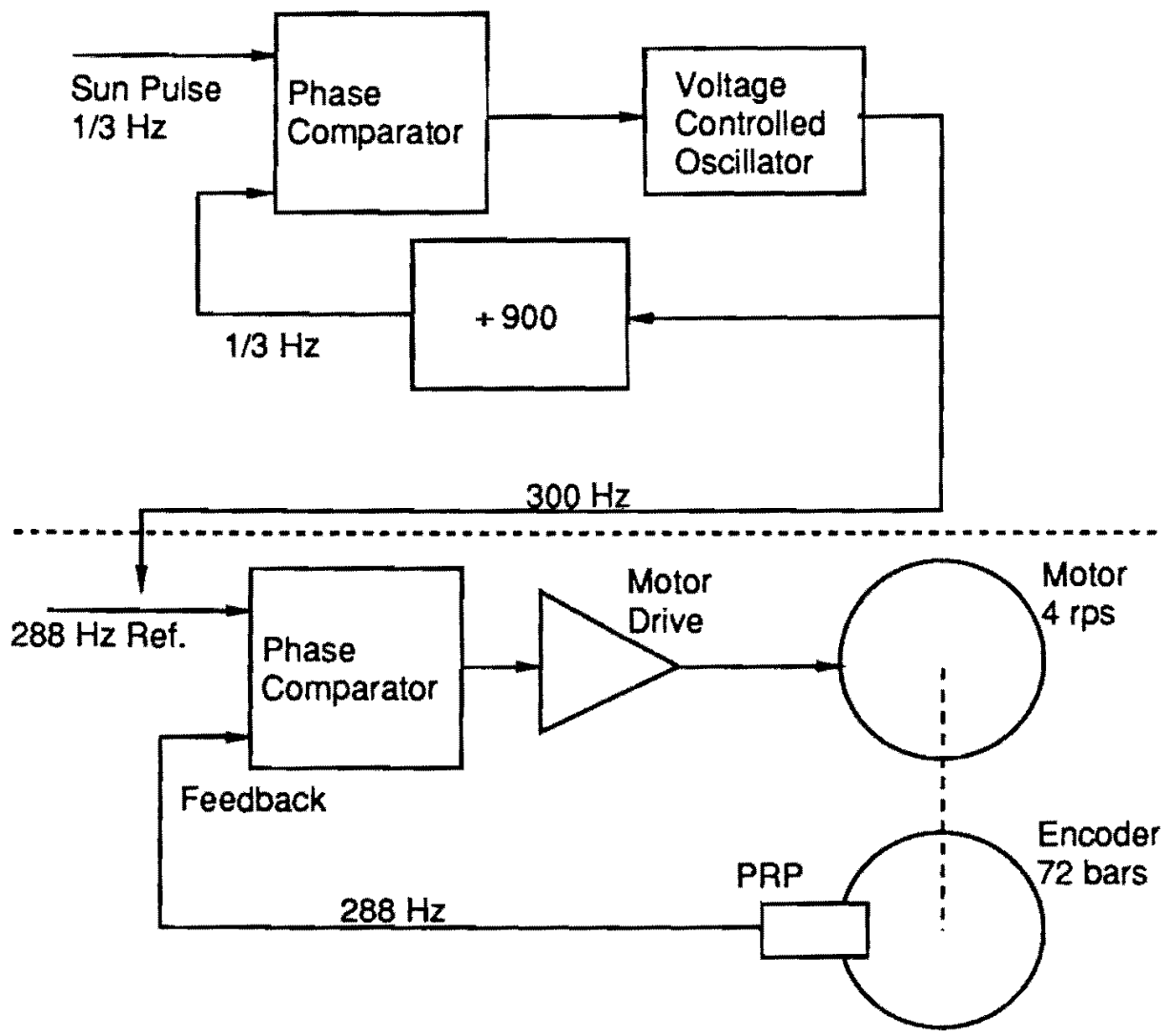

Figure 5: Commandable Rate Scanner Scan Rate Control Schematic (Courtesy of Barnes Engineering) 
Thus for a spacecraft spinning at $20 \mathrm{rpm}$, the reference signal will be $300 \mathrm{~Hz}$, which corresponds to $300 / 72=4.17$ revolutions per second, or $S R=$ 12.51. This arrangement will track the satellite spin rate and maintain the motor speed 12.51 times faster.

Obstructions by spacecraft appendages such as solar arrays or antennas can create mounting difficulties for conical sensors, particularly on three-axis stabilized vehicles. The problem is even more serious when the sensor half-cone angle is large-a $90^{\circ}$ half-cone angle requires a hemisphere of obstruction-free viewing. Fortunately, spinning satellites are generally cylindrical and spacecraft builders tend to keep appendages along the spacecraft spin axis, out of the field of view of the Commandable Rate Scanner. Nevertheless, the CRS half-cone angle can be made smaller if spacecraft appendages are a problem. Of course, as the half-cone angle decreases, a coverage hole develops around the spacecraft spin axis with a radius equal to the complement of the sensor halfcone angle. The coverage hole should be kepr smaller than the the smallest Earth seen by the spacecraft during the mission, unless mission requirements dictate that nadir never be in the vicinity of the spacecraft spin axis.

Table 2 provides a $3 \sigma$ error budget for scanning sensors from Barnes Engineering. The budget is divided into noise and bias sections, with an RSS value of the bias components provided at the bottom of the table.

\section{PROCESSING}

Attitude determination using the Commandable Rate Scanner is performed by measuring multiple horizon crossings over a period of time. Since the disk of the Earth is uniquely defined by three horizon points, an attitude estimate can be obtained after only three horizon measurements are made. However, a principal advantage of the CRS over traditional Earth sensing systems is that the CRS provides multiple horizon crossings, reducing many errors through averaging.

\section{Conversion to Spacecraft Coordinates}

The Commandable Rate Scanner provides horizon crossing times as output. To use these measurements for attitude determination we require a more convenient format. For a planar $\left(90^{\circ}\right.$ half-cone angle) sensor with the sensor spin axis orthogonal to the spacecraft spin axis, the conversion from horizon crossing times to horizon crossing vectors in spacecraft coordinates (at a common epoch) is a simple two-step process.

The angle to the sensor FOV about the spacecraft spin axis from some fiduciary point in inertial space is simply a function of time and the spacecraft spin rate:

\begin{tabular}{|c|c|}
\hline Error Component & $3 \sigma$ Error \\
\hline \multicolumn{2}{|l|}{ Random Errors: } \\
\hline Noise Equivalent Angle for a Single Crossing (240 rpm) & $0.104^{\circ}$ \\
\hline \multicolumn{2}{|l|}{${ }^{2}$} \\
\hline $\begin{array}{l}\text { Earth Radiance Variation } \\
\text { (with 2nd order radiance compensation [ref. 2]) }\end{array}$ & $0.02^{\circ}$ \\
\hline Phase Reference Pickup (PRP) Nonlinearity & $0.005^{\circ}$ \\
\hline Component Aging and Temperature Effects & $0.008^{\circ}$ \\
\hline Alignment Uncertainty & $0.015^{\circ}$ \\
\hline Quantization & $0.002^{\circ}$ \\
\hline Bias RSS & $0.027^{\circ}$ \\
\hline
\end{tabular}

Table 2: Error Budget for Scanning Sensors from Barnes Engineering 


$$
\alpha=\omega_{S / C}\left(t_{H C} \bmod \tau_{S / C}\right)
$$

Similarly, the angle to the sensor FOV from the spacecraft spin plane is simply a function of time and the sensor spin rate:

$$
\delta=\omega_{\text {sensor }}\left(t_{H C} \bmod \tau_{\text {sensor }}\right)
$$

Here $\alpha$ and $\delta$ are, respectively, the horizon crossing azimuth and elevation, in spacecraft coordinates at the epoch time. $\omega_{s / C}$ is the spacecraft spin rate, and $\tau_{S / C}$ is the spacecraft spin period. Likewise, $\omega_{\text {sensor }}$ is the sensor spin rate and $\tau_{\text {sensor }}$ is the sensor spin period. $t_{H C}$ is the horizon crossing time since the epoch. These simple relationships hold because the sensor spin axis is orthogonal to the spacecraft spin axis and because the sensor half-cone angle, $\gamma$, is $90^{\circ}$

Equations for the case when the sensor half-cone angle is not $90^{\circ}$ or when the angle between sensor and spacecraft spin axes are not orthogonal are clearly more realistic. The math is simple to derive, but tedious and not particularly instructive.

When all of the horizon crossing times have been converted to horizon crossing unit vectors, two corrections-for spacecraft motion and Earth oblateness-may be necessary. These corrections are briefly discussed, but implementation details can be found in refs. 3, 4, and 5 .

For most Earth horizon sensing systems, oblateness is the largest error source, particularly at low altitudes, and can contribute as much as several tenths of a degree in attitude error. Oblateness has a much smaller impact on attitude determination using the CRS because the sensor averages a large number of crossings over the entire Earth disk. However, existing oblateness correction algorithms are effective, well-understood, and can be applied to CRS data in exactly the same way as they are applied to data obtained using other sensor types. Oblateness correction algorithms require knowledge of the spacecraft sub-satellite latitude.

Spacecraft motion correction is performed by rotating the horizon crossing vectors about the orbit pole by an amount corresponding to the angular motion of the spacecraft about the Earth. This is particularly simple for a circular orbit, because the size of the rotation angle is directly proportional to the horizon crossing time. A slightly more complex algorithm is needed for elliptical orbits because the angular rate of the spacecraft around the Earth is not constant. The orbit period, phase, eccentricity, and the orbit pole in spacecraft coordinates are needed to correct for spacecraft motion.

\section{Nadir Vector Determination}

As with horizon crossing indicators for spinning satellites, and conical Earth sensors for three-axis stabilized vehicles, we ultimately wish to obtain the direction to nadir. So far, however, we've only obtained a set of horizon crossing unit vectors. An important characteristic of a horizon crossing vector is that the angle between the vector and nadir is the Earth angular radius. Thus,

$$
\mathbf{h} \cdot \mathbf{n}=\cos \rho
$$

where $\mathbf{n}$ is the unit vector to nadir, $\mathbf{h}$ is the horizon crossing unit vector, and $\rho$ is the Earth angular radius. If the altitude is unknown, this equation has four unknowns, three of which are independent ( $\mathbf{n}$ is a unit vector). Therefore, three horizon crossing measurements are sufficient to provide a unique solution to this equation. For $N$ measurements, the equation can be rewritten as

$$
\mathbf{h}_{\boldsymbol{i}} \cdot \mathbf{n}=\cos \rho \quad i=1 . . . N
$$

These equations are more conveniently dealt with in matrix form, so define

$$
\mathbf{H}=\left[\begin{array}{ccc}
h_{1 x} & h_{1 y} & h_{1 z} \\
h_{2 x} & h_{2 y} & h_{2 z} \\
\vdots & \vdots & \vdots \\
h_{N x} & h_{N y} & h_{N z}
\end{array}\right]
$$

and

$$
\mathbf{n}=\left[\begin{array}{l}
\eta_{x} \\
\eta_{y} \\
\eta_{z}
\end{array}\right]
$$


Then eq. 5 becomes

$$
\begin{aligned}
& {\left[\begin{array}{ccc}
h_{1 x} & h_{1 y} & h_{1 z} \\
h_{2 x} & h_{2 y} & h_{2 z} \\
\vdots & \vdots & \vdots \\
h_{N x} & h_{N y} & h_{N z}
\end{array}\right]\left[\begin{array}{l}
\eta_{x} \\
\eta_{y} \\
\eta_{z}
\end{array}\right]=\left[\begin{array}{c}
\cos \rho \\
\cos \rho \\
\vdots \\
\cos \rho
\end{array}\right]} \\
& \mathbf{H n}=\left[\begin{array}{c}
\cos \rho \\
\cos \rho \\
\vdots \\
\cos \rho
\end{array}\right]
\end{aligned}
$$

$\mathbf{H}$ is an $N \times 3$ matrix of horizon crossing unit vector components. We seek $\mathbf{n}$, which is a column vector of nadir vector components. Of course, the CRS may produce hundreds or even thousands of horizon crossings per measurement period. Since only three are needed to solve for the nadir vector, a least-squares solution to an over-determined set of equations is used. More sophisticated algorithms may be used, particularly if measurement errors are well-understood and characterized, but the least-squares method provides excellent results.

The batch least-squares nadir vector determination algorithm is provided primarily for illustrative purposes but also because it was implemented in the CRS simulation discussed below. However, batch algorithms, in general, are inappropriate for CRS processing because they require all of the measurements before a solution can be obtained. For the CRS, this could take as long as several minutes-far too long for control purposes. In addition, this particular algorithm requires multiplication of a $3 \times N$ matrix by its transpose. For simplicity, define

$$
\mathbf{n}^{\prime}=\frac{1}{\cos \rho} \mathbf{n}
$$

so that

$$
\mathrm{Hn}^{\prime}=\mathbf{1}
$$

where 1 is an $N \times 1$ matrix of 1 's. The batch leastsquares estimate for the solution to this equation is

$$
\mathbf{n}_{e s \mathrm{~s}}^{\prime}=\left[\mathbf{H}^{\mathrm{T}} \mathbf{H}\right]^{-1} \mathbf{H}^{\mathrm{T}} \mathbf{1}
$$

This vector points toward nadir and has magnitude $1 / \cos \rho$, so the spacecraft altitude may be obtained obtained from the following relation:

$$
\sin \rho=R_{E} /\left(R_{E}+A l t\right)
$$

Here $R_{E}$ is the Earth equatorial radius and $A l t$ is the spacecraft altitude.

Recursive forms of the least-squares estimator can be found in ref. 6 .

\section{CRS SIMULATOR}

A computer simulation of the Commandable Rate Scanner was developed to assess the system accuracy under various conditions. The simulation is composed of two separate programs: the truth generator and the data processor. The truth generator produces a file of horizon crossing times based on spacecraft orbit and attitude information and sensor geometry. The truth file is then read by the data processor which corrupts the data with noise and biases and computes an attitude estimate.

The truth generator is strictly a kinematic simulation of the spacecraft motion. Spacecraft nutation is included, but the nutation angle must be a constant (i.e., the transverse moments of inertia must be equal). The truth generator requires the following inputs:

- Spacecraft altitude

- Spacecraft spin rate

- Spacecraft principal and transverse moments of inertia

- Nutation angle

- Direction to nadir in spacecraft inertial coordinates

- Direction to the orbit pole in spacecraft inertial coordinates

Sensor mounting information is not an input, but adjustments to the code are simple to make, so modeling sensor biases is easy.

To make the simulation as realistic as possible, the data processor only uses the horizon crossing time and estimates of the spacecraft spin tate, sensor spin rate, an orbit pole estimate, and 
horizon crossing times to produce an attitude estimate. The data processor never uses spacecraft mass properties, nutation, or mounting information, or inputs from the truth generator.

Figure 6 shows a simulation output example when the Earth is $90^{\circ}$ from the spacecraft angular momentum vector. This example includes $0.5^{\circ}$ of nutation as well as noise and bias values of $0.1^{\circ}$ and $0.027^{\circ}$, respectively. The motion of the sensor FOV near the spacecraft spin plane is reflected in this figure where we see a small number of horizon crossings near $+60^{\circ}$ azimuth and $-60^{\circ}$ azimuth, and a large number near $0^{\circ}$ azimuth. In this example the sensor FOV is essentially moving straight up and down the figure.

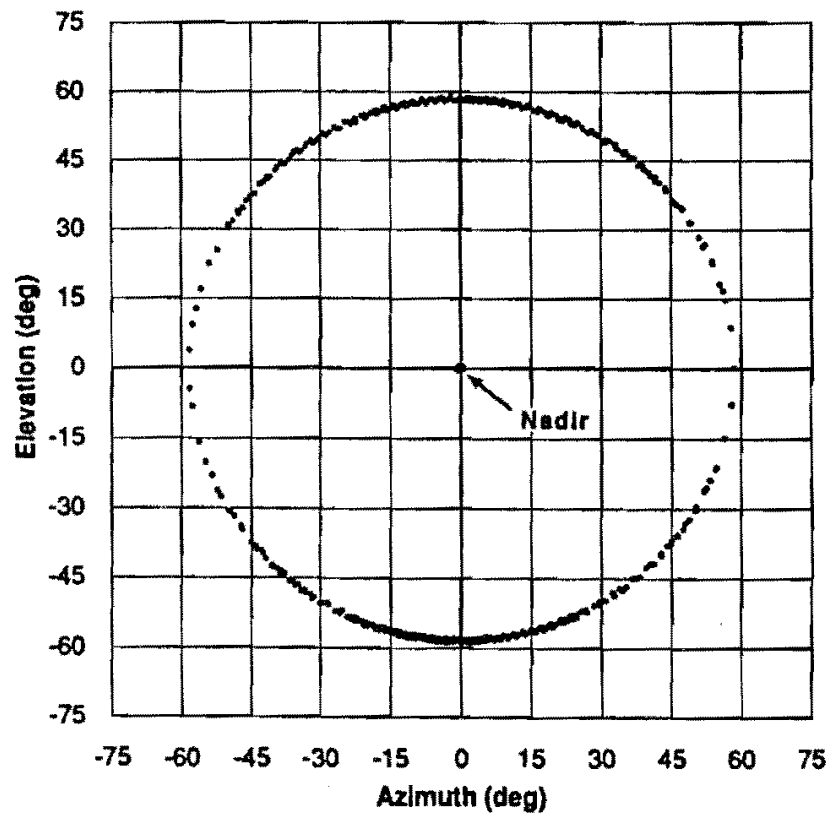

Figure 6: Simulated CRS Data for Earth 90 from the Angular Momentum Vector

In fig. 7 , the spacecraft angular momentum vector is pointing directly at nadir. Here every sensor scan crosses the disk of the Earth, so we obtain a greater number of horizon crossings than in the previous example for the same time span. Also, the horizon crossings are more uniformly distributed around the disk of the Earth. The sinusoidal character of some arcs of data is due to nutation frequencies nearly beating with either sensor or spacecraft spin frequencies; the effect, however, has been found to be negligible.

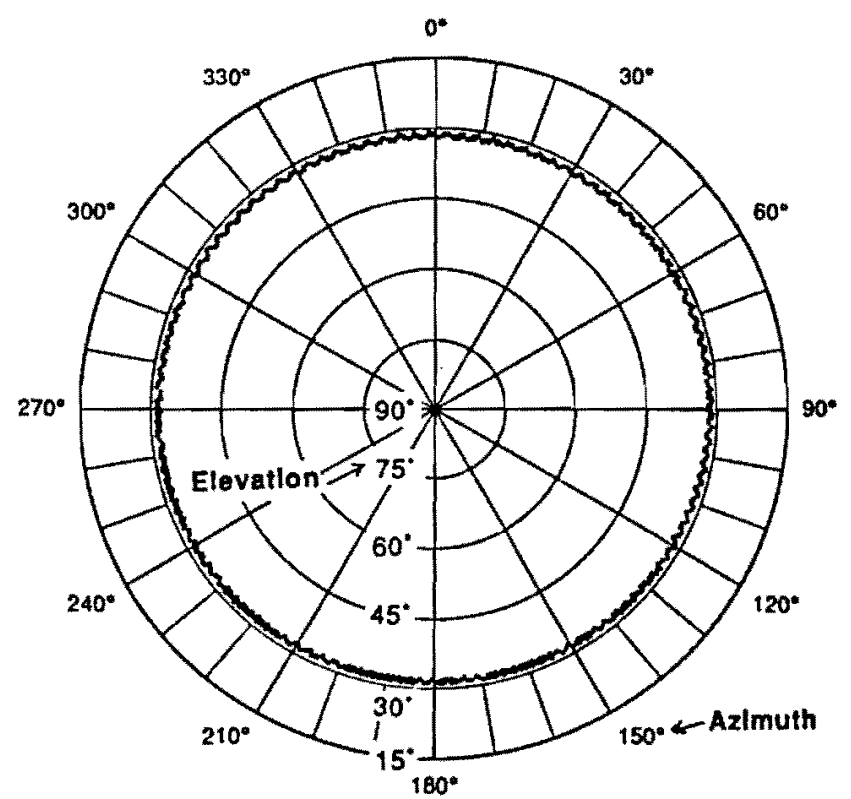

Figure 7: Simulated CRS Data for Earth at the Angular Momentum Vector

The spacecraft altitude in both of these examples was approximately $1100 \mathrm{~km}$, corresponding to an Earth angular radius of about $58^{\circ}$. It is quite clear from figs. 6 and 7 that the Earth angular radius can be accurately determined by averaging the distance between nadir and the horizon crossings.

\section{NUTATION}

To a non-nutating spinning spacecraft, the Earth appears to be traveling around the spacecraft spin axis along a small circle path of constant radius. However, if the vehicle is nutating, the angle between the spacecraft spin axis and the Earth will oscillate sinusoidally with an amplitude equal to the spacecraft nutation angle. The mean radius is, naturally, the radius seen if the spacecraft were not nutating. Nevertheless, this added spacecraft motion directly affects measurements made by almost all attitude sensors, including the $\mathrm{HCI}$ and the CRS [ref. 3].

Eliminating the effects of nutation on attitude sensor data is analytical and computationally complex. Fortunately, this complexity can be avoided with the CRS because enough data can be 
obtained to average out the nutation. Simulation has shown that, over reasonable measurement spans, nutation effects become extremely small. Strictly speaking, nutation is a systematic error which shouldn't be treated as noise. However, a few assumptions can be made which allow us to essentially disregard the systematic character of this error. Specifically, we assume that

- the nutation period is small compared to the measurement time span

- the measurement time span is many nutation periods in length

- the nutation amplitude is small compared to the Earth angular radius

An important question remains regarding nutation. Specifically, if the spacecraft spin axis is not fixed in inertial space, what is the Commandable Rate Scanner solving for? For a nutating spacecraft, the CRS solves for the direction to nadir in a coordinate system defined by the spacecraft angular momentum vector, not the spacecraft spin axis. Equivalently, the CRS measures the angular momentum vector in a coordinate system defined by nadir. The problem remains to find the nutation parameters (amplitude, phase, and frequency) to solve for the spacecraft spin axis relative to the angular momentum vector.

\section{BIASES}

Bias effects and bias determination are of principal importance when high-accuracy attitude sensing is required. A major advantage of the Commandable Rate Scanner over the Horizon Crossing Indicator is that, for the CRS, most biases have little impact on attitude determination accuracy. In addition, they are usually observable from the sensor data alone. Sensor mounting biases are described below and examples are provided. Also note that an uncertainty or misalignment in the orientation of the principal axis in spacecraft coordinates is equivalent to a sensor mounting error. Principal axis translation has no impact on CRS accuracy.
A sensor bias can be categorized as being a phase bias, an azimuth bias, or an elevation bias:

\section{Phase Bias}

A phase bias occurs when the phase reference on the Commandable Rate Scanner is not oriented where expected. Consequently, each horizon crossing will be shifted by a fixed amount relative to where we believe it to be in spacecraft coordinates. A phase bias shifts the horizon in one direction during upward scans, and in the opposite direction during downward scans. Since a large number of both upward and downward scans cross the Earth disk, a "double image" of the Earth results. Phase-biased CRS data can be processed essentially without regard to the bias, because, as the number of horizon crossings grows, the average effect of the bias tends to zero.

Figure 8 shows the effect of a $5^{\circ}$ phase bias on $\mathrm{HCl}$ data. This bias is, of course, unrealistically large and was chosen only for illustrative purposes. Nevertheless, the net effect of this bias after ninety seconds was about $0.05^{\circ}$. A larger, but more realistic phase bias of $0.5^{\circ}$ resulted in $0.01^{\circ}$ residual error after ninety seconds.

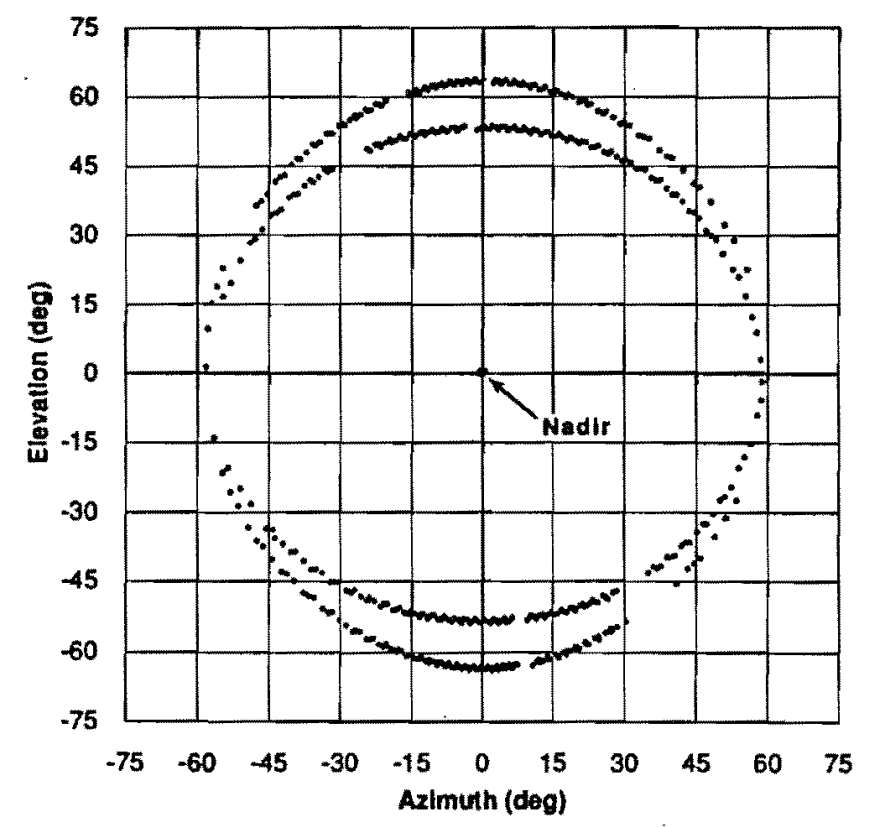

Figure 8: Effect of $5^{\circ}$ Phase Bias. 


\section{Azimuth Bias}

An aximuth bias implies that the angle between the sensor scan axis and a fiduciary mark on the spacecraft (e.g., another sensor, a payload, or an actual mark) is incorrectly known. The impact of an azimuth bias is simply to shift all of the sensor data in azimuth. In other words, if a payload sensor were placed at a different spacecraft azimuth than expected, but the attitude sensor was precisely where it should be, the attitude sensor would be unable to observe the error in the payload sensor orientation. Unfortunately, an azimuth bias is entirely unobservable and produces an irreducible error in the attitude exactly equal to the size of the bias. The effect of a $5^{\circ}$ azimuth bias is shown in Figure 9.

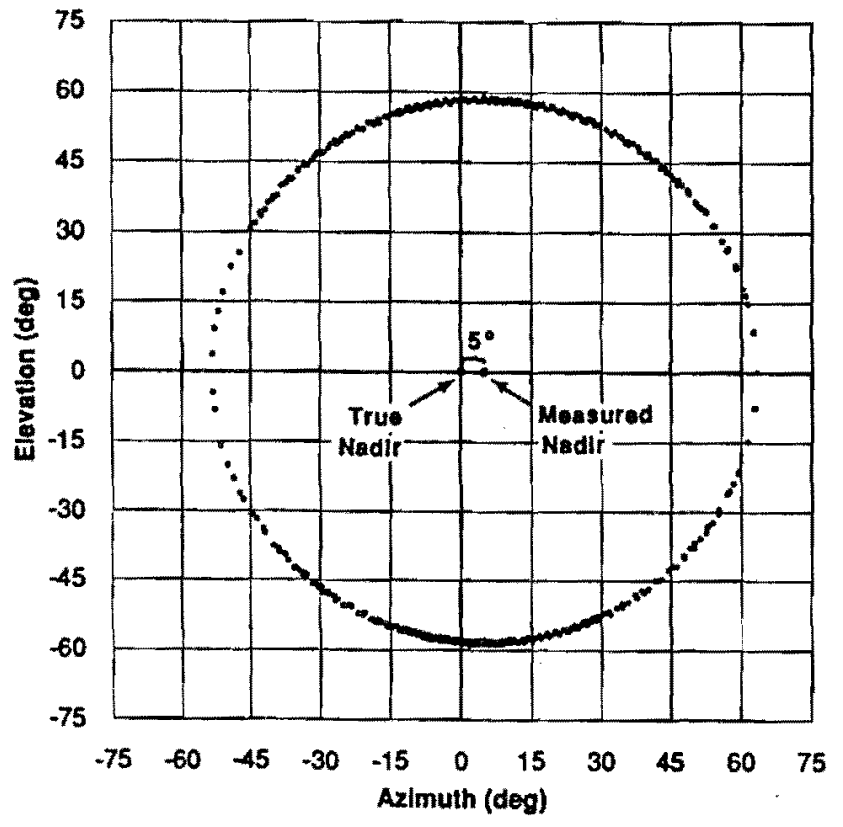

Figure 9: Effect of $5^{\circ}$ Azimuth Bias.

Elevation Bias

An elevation bias results when the sensor scan axis is mounted above or below the spacecraft spin plane. The impact of an elevation bias is similar to that of a phase bias-the horizon crossings will be shifted in one direction for upward scans and in the opposite direction for downward scans. Again, a double image of the Earth will result, allowing for bias detection and determination. Figure 10 illustrates the effect of a $5^{\circ}$ elevation bias on CRS data. Note the differences in the elevation and the phase biases, enabling bias-type discrimination.

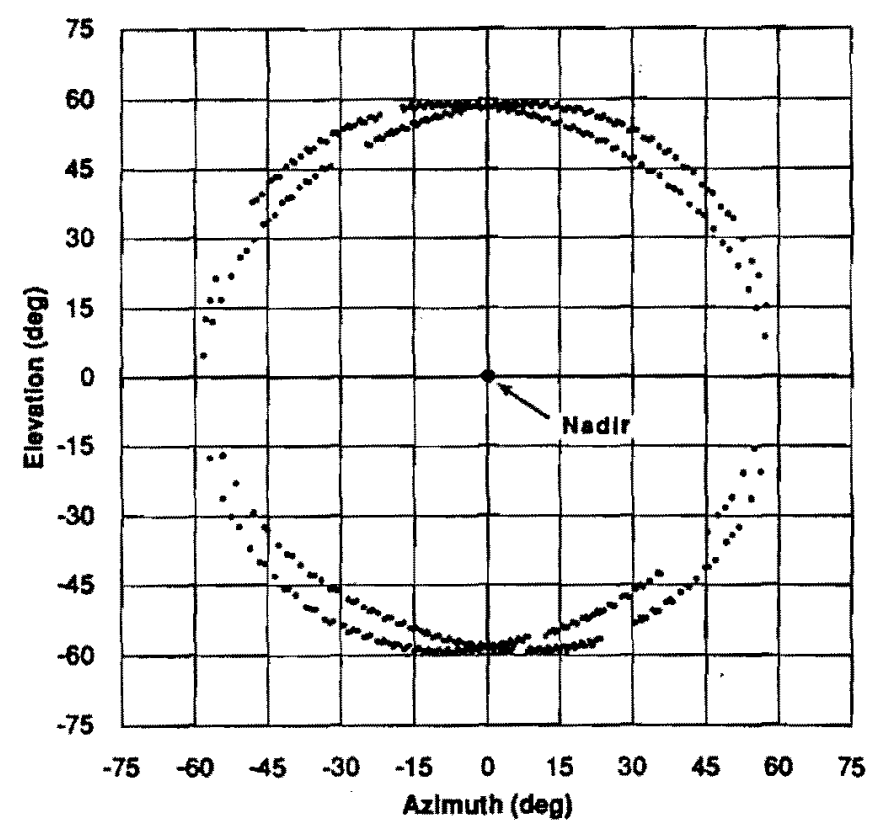

Figure 10: Effect of $5^{\circ}$ Elevation Bias.

\section{PERFORMANCE}

Simulations were run for a $20 \mathrm{rpm}$ spacecraft with a $237 \mathrm{rpm}$ Commandable Rate Scanner in an $1100 \mathrm{~km}$ circular orbit $(S R=11.85)$. The mission geometry is shown in fig. 11. Each run simulated ninety seconds of data and included $0.5^{\circ}$ of nutation. Sensor errors of $0.1^{\circ}$ in noise equivalent angle and $0.027^{\circ}$ in phase bias were also included. Figure 12 plots mean angular momentum vector direction error versus nadir angle (the angle between the nadir vector and the angular momentum vector). Fifty runs were made for each point on the graph. As discussed below, sensitivity to nadir angle was evident; however, mean CRS performance was better than $0.01^{\circ}$ for all cases. The standard deviation was largest at small nadir angles, but was rarely larger than $0.002^{\circ}$, suggesting that the maximum $(3 \sigma)$ angular momentum direction error for the conditions evaluated is about

$$
\begin{aligned}
\text { MaxError } & =0.009^{\circ}+3\left(0.002^{\circ}\right) \\
& =0.015^{\circ}
\end{aligned}
$$


Earth oblateness will degrade sensor performance, but we believe that oblateness corrections can maintain the error contribution to $0.01^{\circ}$ or better, resulting in mean angular momentum vector determination accuracy of better than $0.02^{\circ}$.

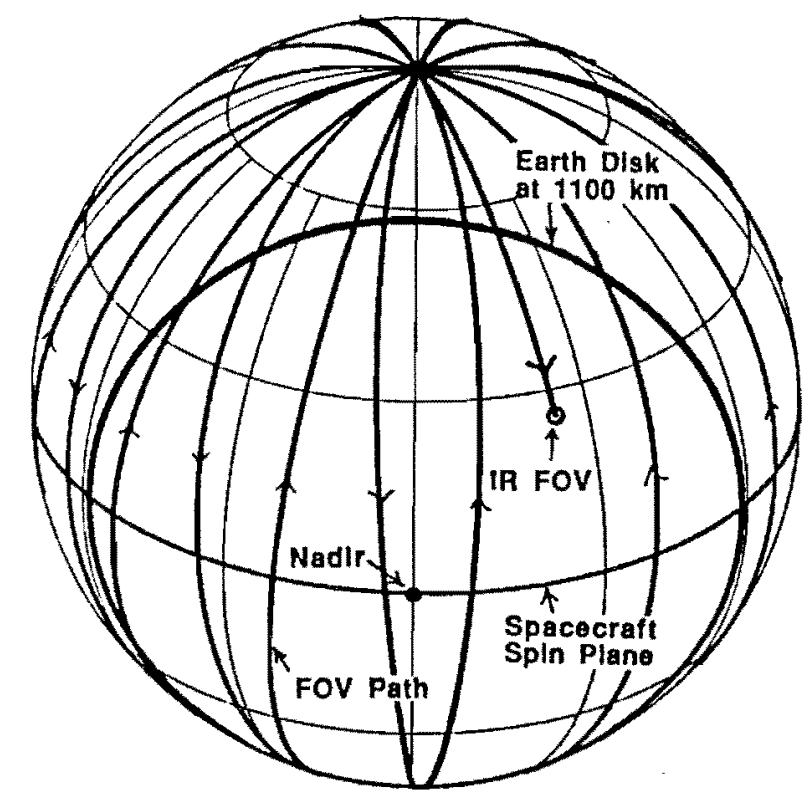

Figure 11: Sensor Sky for Spacecraft at $1100 \mathrm{~km}$ and $S R=11.85$.

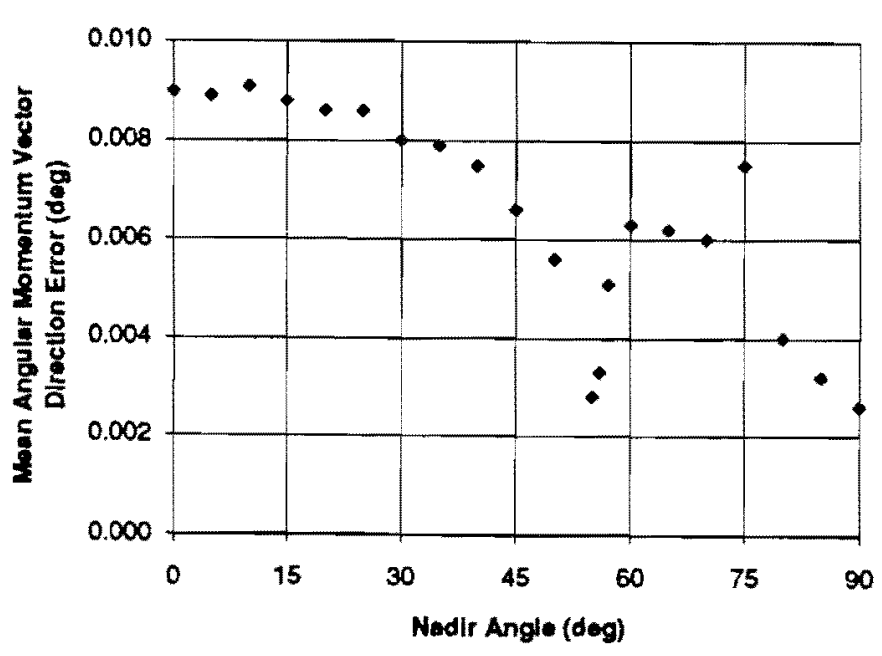

Figure 12: Mean Angular Momentum Vector Determination Error vs. Nadir Angle.

One might reasonably expect to see the best sensor performance when the spacecraft angular momentum vector is pointing at the disk of the Earth (i.e., the nadir angle is smaller than the Earth angular radius) because the highest data rate occurs under this condition. Figure 12 shows, however, that this is not generally true. Indeed, the largest error occurs when the angular momentum vector is pointing directly at, or in the vicinity of, nadir. This can be understood by noting that when the angular momentum vector is pointing at nadir, the sensor errors contribute equally to azimuth and elevation components. On the other hand, when the angular momentum vector is orthogonal to nadir, the majority of the sensor error results in an elevation error-azimuth accuracy is sensitive only to spacecraft spin rate error.

This phenomenon is analogous to sensor gain for conical Earth sensors and horizon crossing indicators. Sensor gain, the derivative of nadir angle with respect to chord width, is a measure of the sensitivity of horizon sensor measurements to horizon sensor errors [ref. 3]. Large gain implies insensitivity and occurs when the sensor scan crosses near the edge of the Earth disk. Similarly, CRS performance improves when some scans cross near the edge of the Earth disk. This occurs often when the angular momentum vector is near the Earth horizon and less often when nadir is near the spacecraft spin plane. As seen in fig. 12 , the performance improvement at a nadir angle of $58^{\circ}$ is followed by a sharp increase in angular momentum vector error because as the angular momentum falls off of the Earth disk, the number of crossings per spacecraft revolution suddenly decreases.

For a non-nutating spacecraft, complete threeaxis attitude determination can be performed using the CRS and a spin phase sensor such as a Sun sensor. However, if the spacecraft is nutating, nutation amplitude, phase, and frequency must also be measured to completely specify the vehicle three-axis attitude.

\section{AUTONOMOUS NAVIGATION}

References 7 and 8 describe a fully autonomous navigation system for three-axis stabilized spacecraft which uses the range to the Earth and the 
directions to the Earth, Sun, and Moon to estimate spacecraft orbit and attitude. Using the method developed therein, it is entirely feasible to perform autonomous navigation for spinning satellites using the Commandable Rate Scanner and an additional Sun/Moon detection system. Given the high data rate of the CRS and the reduced susceptibility to systematic errors, autonav on spinning satellites should outperform autonav on three-axis stabilized spacecraft. Sun sensing can easily be performed using sensors from Adcole, Barnes Engineering, or other sensor manufacturers. Barnes Engineering also produces a visible light sensing system which detects both the Sun and the Moon and can be directly integrated into the Commandable Rate Scanner, thus eliminating intersensor mounting biases.

\section{SUMMARY}

The Cornmandable Rate Scanner-a modified conical Earth sensor-can provide significant advantages over traditional Earth sensors for spinning satellites. The sensor technology is traceable to more than 500 Barnes Engineering scanning infrared sensors and provides continuous, full-sky Earth coverage from LEO to above GEO. The sensor scan pattern can be selected to meet mission needs, and is uniquely characterized by the sensor-to-spacecraft spin ratio, $S R$. A sensor spin rate control circuit keeps $S R$ constant based on spacecraft spin rate measurements from a Sun sensor or other source. Unlike horizon crossing indicators, the CRS is insensitive to significant nutation and sensor biases. Data rates of several hundred measurements per minute in low Earth orbit allow some systematic errors to be ignored, eliminating complex processing algorithms. Angular momentum vector determination accuracy should be better than $0.02^{\circ}$. With Sun and Moon sensing, the Commandable Rate Scanner can provide accurate, fully-autonomous spacecraft attitude and orbit determination.

\section{ACKNOWLEDGEMENTS}

The author would like to acknowledge Dr. James R. Wertz of Microcosm, primarily for coming up with the idea of a spinning sensor on a spinning spacecraft. Credit must also be given to Steve Collins of Microcosm for developing the batch least-squares nadir vector determination algorithm, an idea he hasn't yet published. From Barnes Engineering, Bob Barnes and Bob Astheimer provided technical and managerial support. The Naval Research Laboratory provided funding for much of this work, and Sam Hollander, in particular, provided the initial interest and support, which is greatly appreciated.

\section{REFERENCES}

${ }^{1}$ Wertz, James, R., and Larson, Wiley J., eds., Space Mission Analysis and Design Kluwer Academic Publishers, Dordrecht, Holland, 1991.

${ }^{2}$ Gontin, Richard A., and Ward, Kenneth A., "Horizon Sensor Accuracy Improvement Using Earth Horizon Profile Phenomenology," ALAA Guidance, Navigation, and Control Conference, Monterey, California, August 17-19, 1987.

${ }^{3}$ Wert, James R., ed., Spacecraft Attitude Determination and Controh D. Reidel Publishing Company, Dordrecht, Holland, 1978.

${ }^{4}$ Wertz, James R., and Skulsky, E. David, The Commandable Rate Scanner, Microcosm Report \#910102, January, 1991.

5Collins, Steve, "Oblate Earth Model," Microcosm Technical Memorandum 89/267, August 22, 1989.

${ }^{6}$ Mendel, Jerry M., Lessons in Digital Estimation Theory, Prentice-Hall, New Jersey, 1987.

${ }^{7}$ Tai, Frank, and Noerdlinger, Peter D., "A Low-Cost Autonomous Navigation System," Paper No. AAS 89-001, 12th Annual AAS Guidance and Control Conference, Keystone, Colorado, February 4-8, 1989.

${ }^{8} \mathrm{Ta}$, Frank, and Barnes, Robert, "The Dual Cone Scanner: An Enhanced Performance, Low Cost Earth Sensor", Paper No. AAS 89-013, 12th Annual AAS Guidence and Control Conference, Keystone, Colorado, February 4-8, 1989. 